Proc. of the 11 Int. School on Theoretical Physics Symmetry and Structural Properties of Condensed Matter, Rzeszów 2014

\title{
Measuring of Electric Parameters of Graphene in Presence of Temperature Gradient
}

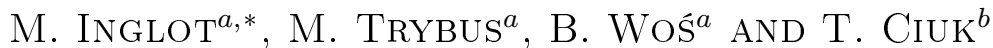 \\ ${ }^{a}$ Department of Physics, Rzeszów University of Technology, al. Powstańców Warszawy 6, 35-959 Rzeszów, Poland \\ ${ }^{b}$ Institute of Electronic Materials Technology, Warsaw, Poland
}

\begin{abstract}
Graphene is one of the most promising materials for application in electronics. It has been recently discovered that on certain substrates graphene is characterized by a rather strong spin-orbit interaction, which is valuable to spintronics applications. In this paper we present an application of a system for measuring of graphene's electrical parameters. The idea of a measuring system originates from the measurements of the pyroelectric coefficient of ferroelectric samples. After a simple modification the system can be used for various experiments related to the determination of electric response of samples exposed to a temperature gradient. Test measurements of monolayer graphene samples and their thermoelectric response are presented.
\end{abstract}

DOI: 10.12693/APhysPolA.128.166

PACS: 85.80.Fi,73.50.Lw, 84.60.Rb

\section{Introduction}

The world currently faces numerous challenges relating to alternative and renewable energy sources. Global demand for the energy increases and at the same time there is a growing concern about the influence of greenhouse gases on the environment. High-efficiency thermoelectric materials are an important group for power generation devices that convert waste heat into electrical energy. The conversion of waste heat into electrical energy seems to be very effective way to improve efficiency of energy production and develop alternative energy technologies reducing our dependence on traditional energy sources. The commercial thermoelectric materials have a relative low figure of merit ZT of 1 and the average thermoelectric generators on the market have a conversion efficiency of approximately $5 \%$ [1].

Our theoretical researches and simulations performed for graphene were very promising regarding efficiency of this material as heat to electricity converter [2].

It was our motivation to conduct practical measurements of thermoelectric effect in graphene samples. Graphene is a plane of carbon atoms [3]. It is the first two-dimensional crystal widely explored in theory and experiment. Discovered about one decade ago, it is still one of the most important and promising material from classical and spin electronics point of view [4-6]. High interest in graphene is mostly due to its electronic properties [7]. Recently, special interest is related to phenomena of thermoelectricity and thermal transport $[8,9]$.

The charge current induced by the temperature gradient is well known for semiconductors and called the Seebeck effect $[10,11]$. The measurable value characterizing thermoelectric properties of any material is the Seebeck

*corresponding author; e-mail: miinglot@gmail.com coefficient. For graphene it was theoretically calculated as giant value equal to $30 \mathrm{mV} / \mathrm{K}$ [12].

Thermoelectric power and electrical conductivity in a wide range of temperatures were studied in a graphene device with a different impurity concentration by Wang and Shi [13]. The influence of strong impurity scattering on the thermoelectric properties on graphene was considered theoretically using self-consistent $t$-matrix approach by Lofwander and Fogelstrom [14]. The thermoelectric properties of graphene in the presence of magnetic field and external disorder describing by Dirac Hamiltonian for low energy excitation was also considered [15, 16].

Electric and thermoelectric transport for the nonzero value of the Rashba spin-orbit interaction was discussed by Alomar and Sánchez [17]. Authors predicted that this effect can be used for detection of nonhomogeneous spinorbit interactions. The effect of substrate in graphene negatively influences and reduces the phonon thermal conductivity, which is desirable feature in physics of thermoelectric effects [18]. The heat flux and electronphonon thermal conductance in disordered graphene sheet was calculated using the Fermi golden rule by Wei et al. [19].

From the presented calculations it follows that the thermal conductance in graphene depends on disorder and screening. It also reflects the underlying chiral nature of electrons in this material. Obtained theoretical results are promising enough to undertake efforts for experimental verification [2].

\section{Experiment}

Measurements of thermoelectric current were performed for graphene layers deposited on two different substrates - namely on $\mathrm{SiC}$ and $\mathrm{SiO}_{2}[20]$. Monolayer chemical vapor deposition (CVD) graphene grown on copper foil and transferred onto conductive silicon substrate $(10 \mathrm{~mm} \times 10 \mathrm{~mm})$ covered with $300 \mathrm{~nm}$ thermal silicon oxide layer was purchased at Nano Carbon Sp. z o.o. 
The Hall effect characterisation proved hole concentration $n=+8.614 \times 10^{12} \mathrm{~cm}^{-2}$, hole mobility $\mu=$ $1415 \mathrm{~cm}^{2} /(\mathrm{V} \mathrm{s})$ and sheet resistance $R=512 \Omega / \mathrm{sq}$, all measured in van der Pauw geometry with the four probe needles placed in the four corners of the sample.

The second investigated sample was epitaxial monolayer graphene grown on the $\mathrm{Si}$ face of a semi-insulating on-axis $4 \mathrm{H}-\mathrm{SiC}(0001)(10 \mathrm{~mm} \times 10 \mathrm{~mm})$ substrate using the epitaxial chemical vapor deposition method in a standard hot-wall CVD Aixtron VP508 reactor [21]. Prior to growth, in situ etching of the $\mathrm{SiC}$ surface was carried out in hydrogen atmosphere. The epitaxial CVD growth of graphene was realized under dynamic flow conditions that simultaneously inhibit silicon atom sublimation and promote the mass transport of propane molecules to $\mathrm{SiC}$ substrate. The as grown sample was characterized by a Hall effect measurement (with the use of an 0.55T Ecopia HMS-3000 setup) in the van der Pauw geometry with the four golden probes placed in the corners of the $10 \mathrm{~mm} \times 10 \mathrm{~mm}$ substrate. Graphene was found to exhibit electron concentration $n=-9.08 \times 10^{12} \mathrm{~cm}^{-2}$, electron mobility $\mu=571 \mathrm{~cm}^{2} /(\mathrm{V} \mathrm{s})$ and sheet resistance $R_{\mathrm{S}}=1116 \Omega / \mathrm{sq}$.

Block diagram of the measurement system setup is presented in Fig. 1a. Carbon back electrodes were applied to the samples in order to limit the influence of electric contacts placed in different temperatures $\left(T_{1}, T_{2}\right)$. Bottom sides of the graphene samples (Fig. 1c) were covered by thin layer of graphite. Such created thermopile minimized the thermoelectric input of copper electrodes of sample applicator. Graphene samples were placed inside the thermostatic unit with use of chosen sample applicator. Two micro Peltiers cells were used for controlling the temperature $T_{1}$ and $T_{2}$ of both sides of the investigated sample. Application of micro Peltier cells made possible independent cooling and heating of both sides of the sample. Thus the high temperature gradient $\nabla T$ inside the sample could be obtained. Two temperature sensors were mounted right under the surface of the heaters (very close to the surface of the samples). Control of the temperature was realized by voltage and current manipulation realized by the software and applied interfaces.

Cross-section of the sample holders is presented in Fig. 1b. Cross-sections of the sample holder applicators are given in Fig. 1c and d. Two different sample applicators were used to determine the influence of the copper electrodes on thermoelectric response of the samples. After preliminary tests sample applicator presented in Fig. 1c was chosen for further measurements.

Thermoelectric current $j$ of the short circuited samples was measured for temperature difference $\Delta T$ applied to the samples. The variation of temperature is presented in Fig. 2a,b. LAB-VIEW virtual interface was designed and created in order to record, process and present measurement data [22]. The thermoelectric current $j$ was measured in room temperature $T=300 \mathrm{~K}$ and air atmosphere. From simple theoretical approach the electric current in case of zero electric field $E=0$ and
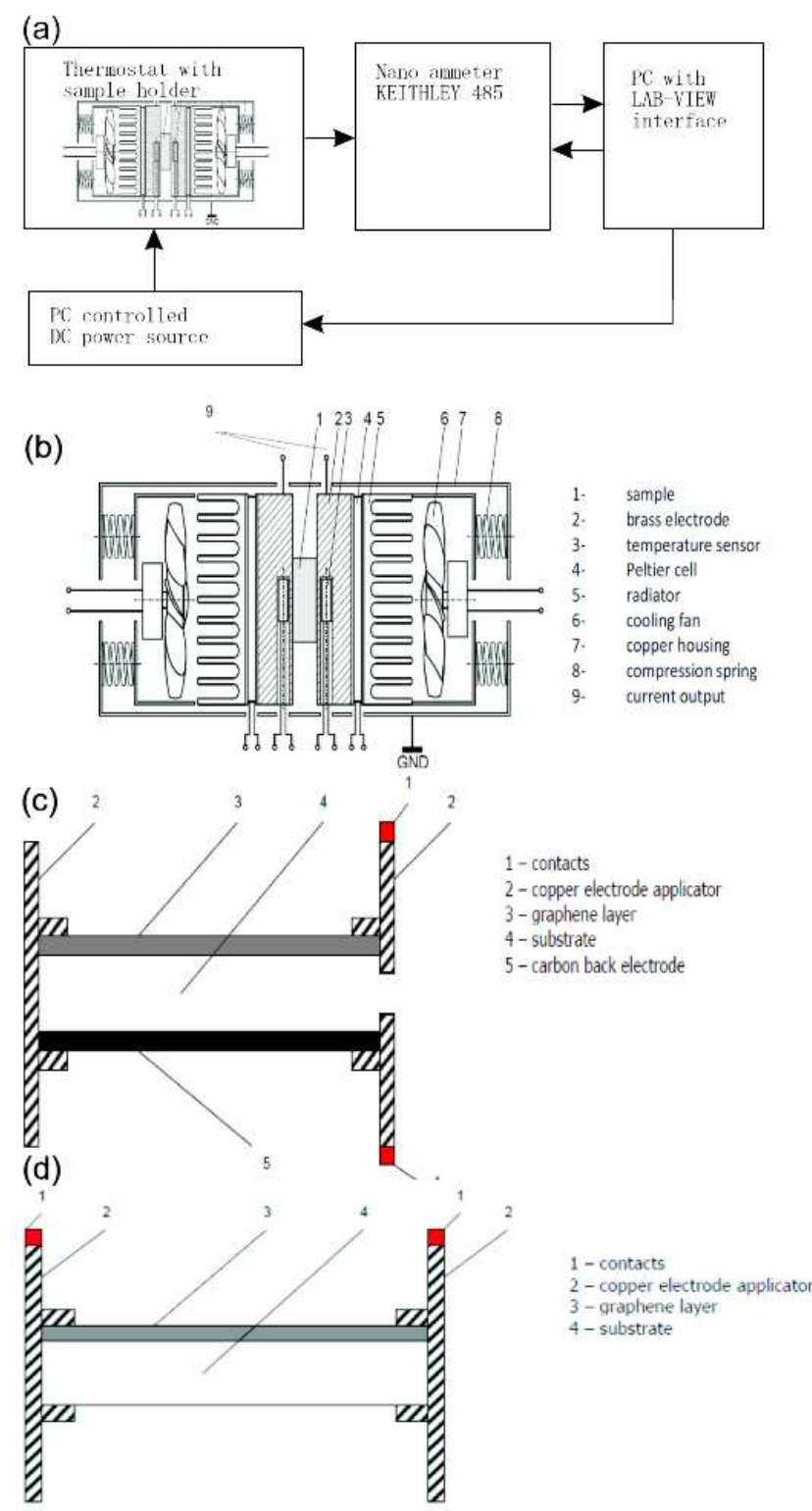

Fig. 1. (a) Block diagram of measuring system, (b) cross section of the sample holder, (c) sample applicator with compensated contact influence, (d) regular sample applicator [22].

homogeneous chemical potential $\nabla \mu=0$ can be written as $j=\sigma \alpha \nabla T$ and it is a linear function of $\nabla T$, where $\sigma$ is the electron conductance and $\alpha$ the Seebeck coefficient in graphene. We measured the diffusion thermoelectric current induced by temperature gradient as function of time $t$ and temperature difference $\Delta T$. The change of temperature with time $t$ for two different graphene samples, described above as $\mathrm{SiC}$ and $\mathrm{SiO}_{2}$, is presented in Fig. 2a. Recording of $\Delta T$ was started from two different temperature levels for each sample.

Initial temperature for graphene on $\mathrm{SiC}$ was approximately $\Delta T \approx 40^{\circ} \mathrm{C}$, while for $\mathrm{G} / \mathrm{SiO}_{2}$ it was $\Delta T=0$. The sign of $\Delta T$ was positive for $T_{2}>T_{1}$ and negative for the opposite. The sign of $\Delta T$ is positive for $T_{2}>T_{1}$ 

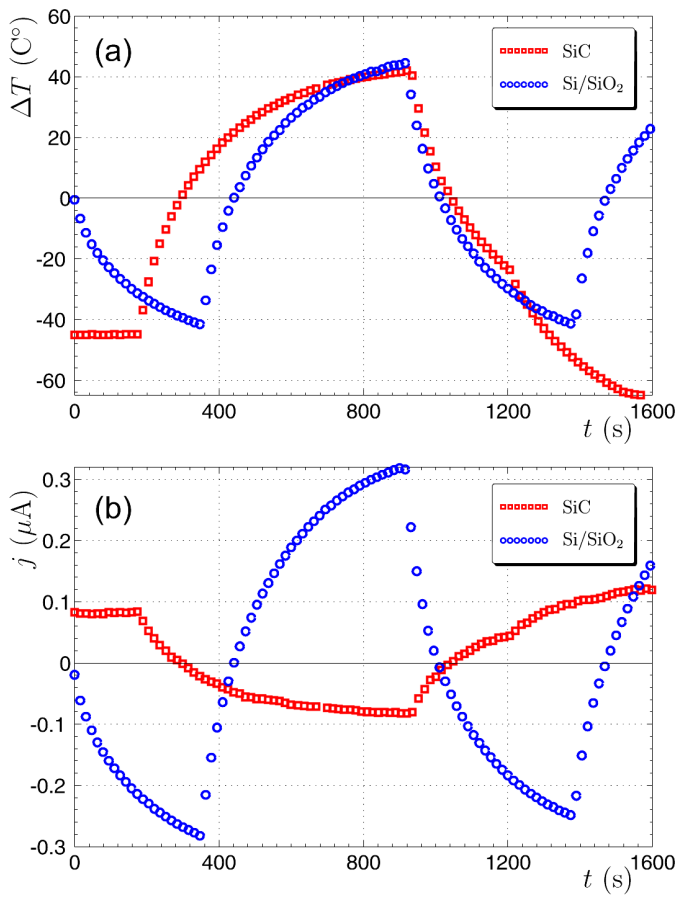

Fig. 2. (a) The difference of temperature $\Delta T$ as a function of time $t$. (b) Current $j(\mu \mathrm{A})$ induced by temperature difference $\Delta T$ as a function of time $t$. The red color refers to monolayer graphene on $4 \mathrm{H}-\mathrm{SiC}$, whereas the blue color to graphene on $\mathrm{Si} / \mathrm{SiO}_{2}$.

and negative on the other case $T_{2}<T_{1}$. For both presented samples the tendency in changes of $\Delta T$ sign with time is similar. Time dependence of current response of tested material to the temperature gradient can be observed in Fig. 2b. The constant value of temperature $\Delta T \approx 40^{\circ} \mathrm{C}$ is manifested by constant electrical current induced by these temperature conditions presented in Fig. 2b. Note that the sign of current is related to majority charge-free electron or hole.

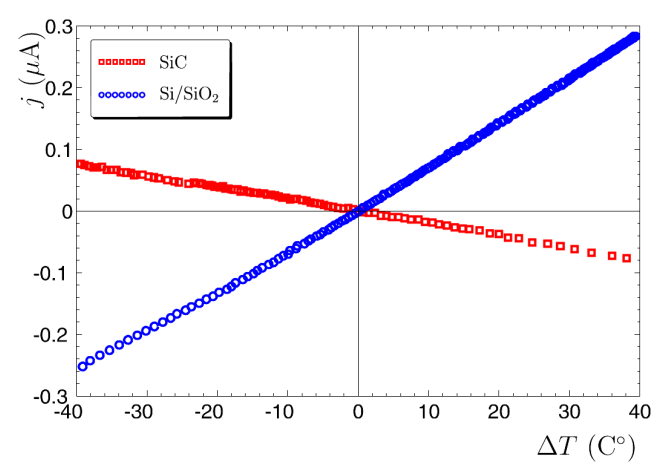

Fig. 3. Thermoelectric current $j$ as function of temperature difference $\Delta T$ for this same samples presented in Fig. 1.

The recorded values of thermoelectric current are presented in Fig. 3. Basically, as one can see, the increase of $\Delta T$ parameter involves the increase of thermoelectric current $j$, as shown in Fig. 2b. The recorded values of thermoelectric current are presented in Fig. 3. Linear character of thermoelectric response of the measured samples confirms theoretical predictions for the semiclassical Boltzmann approach [2].

Two different plots of electric current vs. $\Delta T$ are presented in Fig. 3. Both currents are equal to zero $j=0$ when $\Delta T=0$ which means that move of electrons is thermally excited. We discuss two graphene samples in which majority charge sign and concentration are different. Red squares in Fig. 3 represent the current $j$ for graphene on $\mathrm{SiC}$ substrate where electrons are responsible for conduction. Small concentration of free electrons $n_{\mathrm{e}}=-9.08 \times 10^{12} \mathrm{~cm}^{-2}$ is manifested by small angle of red curve. Opposite situation is shown by blue circles which are associated with graphene on $\mathrm{Si} / \mathrm{SiO}_{2}$ where majority charge are holes of concentration $n_{\mathrm{p}}=+5.98 \times 10^{12} \mathrm{~cm}^{-2}$.

\section{Conclusions}

Graphene samples used in our experimental studies were obtained with the use of two different techniques, namely epitaxial chemical vapor deposition on silicon carbide from a propane precursor and chemical vapor deposition on copper foil from a propane precursor followed by a transfer onto the target substrate through the PMMAmediated electrochemical delamination method. This two materials are characterized by two different conduction charges which can be clearly observed in Fig. 3 where the same temperature gradient $\nabla T$ direction results in opposite direction of measured thermoelectric current. Current flow was reversed in case of opposite direction of temperature gradient. Electric current growth rate is proportional and determined by charges concentration.

After the experiment recorded data, after processing, were compared with theoretical simulation. Performed measurements are in good agreement with model results based on the Boltzmann kinetic equation [2]. These results are very promising for our future researches both within theoretical and experimental researches.

\section{Acknowledgments}

The work of M.I. is partly supported by the projects Nos. DEC-2011/01/N/ST3/00394 and DEC2012/06/M/ST3/00042. The work of T.C. is supported by National Science Centre in Poland under the PRELUDIUM 2013/11/N/ST3/04147 grant.

\section{References}

[1] Y.C. Lan, A.J. Minnich, G. Chen, Z.F. Ren, Adv. Funct. Mater. 20, 357 (2010).

[2] M. Inglot, A. Dyrdal, V.K. Dugaev, J. Barnaś, Phys. Rev. B 91, 115410 (2015).

[3] M.I. Katsnelson, K.S. Novoselov, A.K. Geim, Nat. Phys. 2, 620 (2006). 
[4] M. Inglot, V.K. Dugaev, E.Ya. Sherman, J. Barnaś, Phys. Rev. B 89, 155411 (2014).

[5] F. Donati, L. Gragnaniello, A. Cavallin, F.D. Natterer, Q. Dubout, M. Pivetta, F. Patthey, J. Dreiser, C. Piamonteze, S. Rusponi, H. Brune, Phys. Rev. Lett. 113, 177201 (2014).

[6] E.I. Rashba, Phys. Rev. B 79, 161409 (2009).

[7] K.S. Novoselov, A.K. Geim, S.V. Morozov, D. Jiang, M.I. Katsnelson, I.V. Grigorieva, S.V. Dubonos, A.A. Firsov, Nature 438, 197 (2005).

[8] Z. Aksamija, I. Knezevic, Phys. Rev. B 90, 035419 (2014).

[9] Z.G. Fthenakis, Z. Zhu, D. Tomanek, Phys. Rev. B 89, 125421 (2014).

[10] L. Liang, E. Cruz-Silva, E. Costa Girão, V. Meunier, Phys. Rev. B 86, 115438 (2012).

[11] S. Yig̃en, V. Tayari, J.O. Island, J.M. Porter, A.R. Champagne, Phys. Rev. B 87, 241411(R) (2013).

[12] D. Dragoman, M. Dragoman, Appl. Phys. Lett. 91, 203116 (2007).

[13] D. Wang, J. Shi, Phys. Rev. B 83, 113403 (2011).
[14] T. Lofwander, M. Fogelstrom, Phys. Rev. B $\mathbf{7 6}$, 193401 (2007).

[15] Y.M. Zuev, W. Chang, P. Kim, Phys. Rev. Lett. 102, 096807 (2009).

[16] X.Z. Yan, C.S. Ting, Phys. Rev. B 80, 165423 (2011).

[17] M.I. Alomar, D. Sánchez, Phys. Rev. B 89, 115422 (2014).

[18] J.H. Seol, I. Jo, A.L. Moore, L. Lindsay, Z.H. Aitken, M.T. Pettes, X. Li, Z. Yao, R. Huang, D. Broido, N. Mingo, R.S. Ruoff, L. Shi, Science 328, 213 (2010).

[19] P. Wei, W. Bao, Y. Pu, C.N. Lau, J. Shi, Phys. Rev. Lett. 102, 166808 (2009).

[20] J. Borysiuk, R. Bożek, W. Strupiński, A. Wysmołek, K. Grodecki, R. Stępniewski, J.M. Baranowski, J. Appl. Phys. 105, 023503 (2009).

[21] W. Strupinski, K. Grodecki, A. Wysmolek, R. Stepniewski, T. Szkopek, P.E. Gaskell, Nano Lett. 11, 1786 (2011).

[22] M. Trybus, W. Proszak, B. Woś, Infrared Phys. Technol. 61, 81 (2013). 